\title{
Rheological investigations of food gels under high pressure
}

\author{
Kulisiewicz, L. ${ }^{*}$, Baars, A., Delgado A.
}

Lehrstuhl für Strömungsmechanik, Technische Fakultät, Friedrich-Alexander Universität Erlangen-Nürnberg, Cauerstr. 4, 91058 Erlangen, Germany

leszek.kulisiewicz@1stm.uni-erlangen.de

* Corresponding author

Keywords: high pressure, gelatin gel, rheology, in-situ measurements

\section{Introduction}

Application of high hydrostatic pressure up to $1 \mathrm{GPa}$ offers a variety of advantages and potentials in the modern technology of food processing. It enables inactivation of microorganisms or enzymes while the amount of aromas and vitamins is not substantially decreased [Sasagawa and Yamazaki, 2002]. However, physical properties of the biomatter change significantly due to increase of pressure [Kulisiewicz et al. 2006]. The knowledge of physical properties of treated substance is essential for understanding, design and control of the processing technology. Specifically rheological data are from elevated interest as they provide information on flow behaviour and molecular structure of the investigated substance. Numerous food systems, for which high pressure technology might potentially bring significant advantages [Suzuki, 2002], feature viscoelastic behaviour (e.g., jams, fruit jellies, pastes, creams, yoghurt). A prevalent component of those foodstuffs is gelatin. This animal origin protein is used as a gelling agent, stabiliser, thickener and texturizer [Guo, 2003]. Gelatin plays an important role in production of foods with reduced fat content to simulate the mouth feel of fat without extensive calories.

The present contribution reports on rheological investigations of household gelatine gels under high pressure. Since the proper measurement technique is missing, for the purpose of this study an in-situ rheometer was developed for non-destructive investigations of viscoelastic substances under pressure up to $300 \mathrm{MPa}$.

\section{Materials and Methods}

Experimental setup. The measurement system developed within the present study uses transmission of small amplitude torsional shear waves for determination of storage and loss moduli G' and G'. The test is non destructive, allowing investigations of shear sensitive samples, e.g., critical gels. The sample fills the gap between two parallel plates of $23 \mathrm{~mm}$ diameter, each plate connected to a piezoelectric element, one working as the sensor, one as the actor. High pressure vessel containing the developed rheometer can stand pressures up to $300 \mathrm{MPa}$. Temperature of the sample can be regulated by a glycol bath surrounding the pressure vessel. A thermocouple placed inside the sample chamber provides one-point measurement of the sample temperature.

Sample preparation and experimental procedure. Gelatin solution is used as a model system for preliminary tests. The sample is prepared according to the method given in [Djabourov et al., 1988] as a $0,1 \mathrm{w} / \mathrm{w}$ household gelatin aqueous solution. The solution is poured into the measurement chamber of the rheometer at $40^{\circ} \mathrm{C}$. Next, the rheometer is placed inside the pressure cell and the pressure is increased up to $100 \mathrm{MPa}$. After pressure increase the sample is held at $40^{\circ} \mathrm{C}$ for 30 minutes in order to equilibrate the temperature inhomogeneities developed due to polytropic compression. Afterwards, the temperature of the thermal bath is set to $20^{\circ} \mathrm{C}$. Due to large thermal inertia of the pressure chamber, it takes around 25 minutes 
until the quench temperature of $20^{\circ} \mathrm{C}$ is reached inside the chamber. The measurement is being carried out for 300 minutes. After that period the temperature is increased again up to $40^{\circ} \mathrm{C}$. The chamber is held at that temperature for several hours followed by a pressure increase up to $200 \mathrm{MPa}$. After the equilibration time, temperature is decreased again to $20^{\circ} \mathrm{C}$ and the measurement is started

\section{Results and Discussion}

The first results on the development of the storage modulus $G$ ' over time under high pressure are depicted in Fig. 1. The measurement at atmospheric conditions and comparative measurement at dynamic stress rheometer (Rheometric Scientific SR-5000) used for means of calibration are shown as well. The time is referred to the total measurement time $t_{\text {end }}$ and the storage modulus to the value at $t=t_{\text {end }}$. The first measurements carried out at 100 and 200 MPa reveal that gelatin has kept its jellifying properties under high pressure.

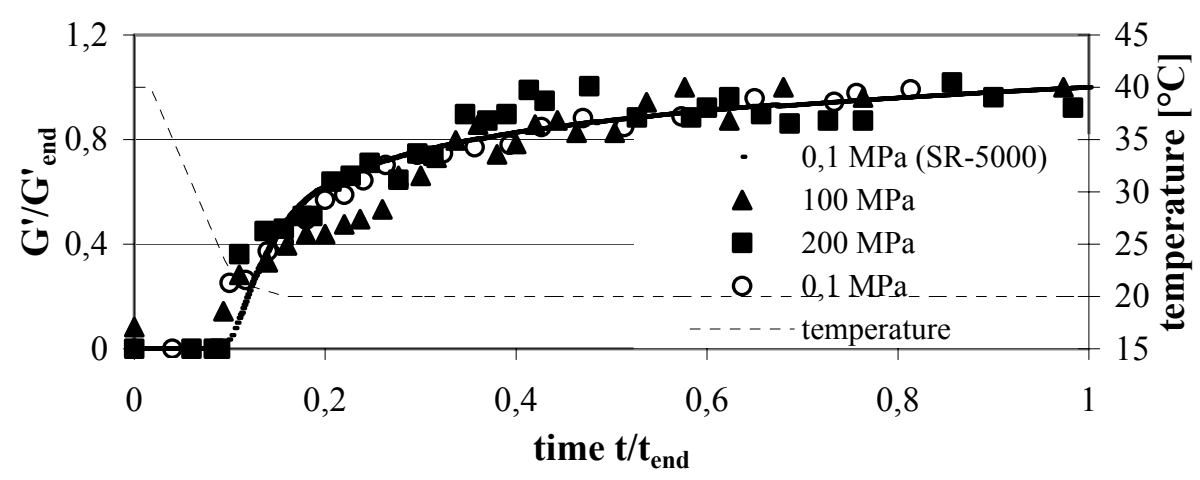

Fig. 1: Development of the storage modulus $G^{\prime}$ over time during gelation of gelatine

The storage modulus at the end of experiment at ambient pressure equals $3,8 \mathrm{kPa}$, at $100 \mathrm{MPa}$ reaches $8 \mathrm{kPa}$ and $15 \mathrm{kPa}$ at $200 \mathrm{MPa}$. Apparently, the storage modulus of gelatine gels is increasing with pressure. However, as it can be seen from the relative representation in Fig. 1, the course of gelation curves is similar for all investigated pressure values, which suggests similar nature of the gelation process. It is hypothesised that the reason for higher values of storage modulus is the higher number of triple helix junction zones along each gelatin molecular strand. The triple helix junction zones connecting three molecular strands are stabilised by hydrogen bonds. The stable length of a junction zone depends on the strength of hydrogen bonds [Guo, 2003], being shorter when hydrogen bonds exhibit higher stability. This is the case under elevated pressure [Heremans and Smeller, 1998], which should lead to a decrease of triple helix junction zone length. Since the storage modulus of gelatin gel is increasing, it is concluded that the reason is the higher number of junction zones per molecular strand. However, the corroboration of the presented here hypothesis requires further investigations.

\section{References}

Djabourov, M., Leblond, J., Papon, P. (1988) Journal de Physique 49, 319

Guo, L. (2003) Ph.D.-thesis, Pennsylvania State University

Heremans K., Smeller L. (1998) Biochimica et Biophysica Acta 1386, 353

Kulisiewicz L., Baars. A., Delgado A. (2006) Proceedings $3^{\text {rd }}$ International Meeting on High Pressure Chemical Engineering, Erlangen, 10.-12. Mai 2006, 103

Sasagawa, A., Yamazaki, A. (2002) In: Trends in High Pressure Bioscience and Biotechnology, Hayashi, R. (ed.), Elsevier Science, Amsterdam, 375-384

Suzuki, A. (2002) In: Trends in High Pressure Bioscience and Biotechnology, Hayashi, R. (ed.), Elsevier Science, Amsterdam, 365-374 